\title{
CARLEMAN INEQUALITIES FOR THE DIRAC OPERATOR AND STRONG UNIQUE CONTINUATION
}

\author{
YONNE MI KIM \\ (Communicated by Barbara L. Keyfitz)
}

\begin{abstract}
Using a Carleman inequality, we prove a strong unique continuation theorem for the Schrödinger operator $D+V$, where $D$ is the Dirac operator and $V$ is a potential function in some $L^{p}$ space.
\end{abstract}

\section{INTRODUCTION}

Let $U$ be a nonempty connected open subset of $R^{n}$, and $u$ be a solution of the differential equation

$$
(D+V) u=0 .
$$

Here $D$ is the Dirac operator and $V \in L^{s}\left(R^{n}\right)$ for some suitable $s$. The main theorem says if $u$ vanishes to infinite order at a point, then $u=0$ identically. This is called a unique continuation theorem because it says that the behavior of a solution at a point determines the behavior in a neighborhood. In 1939 Carleman [2] proved this theorem when $n=2$ and $V$ is bounded, and all subsequent work follows his basic idea. The main step is to prove Carleman inequalities. We need the following type of inequality:

$$
\left\|e^{t \phi} \nabla f\right\|_{L^{q_{1}(U \backslash\{0\}, d x)}} \leq C\left\|e^{t \phi} \Delta f\right\|_{L^{p}(U \backslash\{0\}, d x)}, \quad f \in C_{0}^{\infty}(U \backslash\{0\}) \frac{1}{p}-\frac{1}{q_{1}}=\frac{1}{r},
$$

for $C$ independent of $t$ as $t \rightarrow \infty$ and $U$ an open neighborhood of the origin, where $\phi$ is a suitable weight function which is radial and decreasing. Once this inequality is proved, a straightforward argument due to Carleman yields uniqueness. The key feature that distinguishes these inequalities from ordinary Sobolev inequalities is that the constant $C$ is independent of the parameter $t$. Our main contribution is to improve an earlier unique continuation theorem due to Hörmander [3] or Jerison [4] which required that $u$ vanish on an open set rather than at a single point. In particular Hörmander proved inequalities of type (1) in the special case in which the function $f$ vanishes not only at the origin, but also in a ball $B$ about the origin of fixed positive radius. There was a great deal of work going on and references can be found in $[3,10]$. In order to obtain optimal inequalities of type (1) with a radial decreasing weight

Received by the editors July 2, 1993 and, in revised form, October 19, 1993.

1991 Mathematics Subject Classification. Primary 35J10, 35R45.

(C) 1995 American Mathematical Society 
function, we have to choose $\phi$ carefully. We will use the weight function $\phi$ defined implicitly by $\phi(x)=\psi(y), y=-\psi(y)+e^{-\varepsilon \psi(y)}$ when $y=\log |x|<0$. The idea is from Alinhac-Baouendi [1]. Then $e^{t \phi} \sim|x|^{-t}$. This is an algebraic blowup but still can be handled since $u$ vanishes to infinite order at the origin. This is better than $|x|^{-t}$ because of convexity: $(\partial \psi / \partial y)^{2} \geq e^{\varepsilon y}$.

\section{Statements of Results}

The Dirac operator is a first-order constant coefficient operator on $R^{n}$ of the form $D=\sum_{j=1}^{n} \alpha_{j} \partial / \partial x_{j}$, where $\alpha_{1}, \ldots, \alpha_{n}$ are skew hermitian matrices satisfying the Clifford relations: $\alpha_{j}^{*}=-\alpha_{j}$ and $\alpha_{j} \alpha_{k}+\alpha_{k} \alpha_{j}=-2 \delta_{j k}, j, k=$ $1, \ldots, n$. Also $D^{2}=-\Delta$ and Carleman estimates for $D$ imply estimates of type (1).

Let $\phi(x)=\psi(y)$, be defined as above.

Theorem 1. Let $n \geq 3$. There is a constant $C$ depending only on $n$ such that for all $t \in R$ and for all $h \in C_{0}^{\infty}((-\infty, 0) \times S)$

$$
\sqrt{t}\left\|e^{t \phi} h\right\|_{L^{2}((-\infty, 0) \times S, d y d w)} \leq C\left\|e^{t \phi} D h\right\|_{L^{2}((-\infty, 0) \times S, d y d w)} .
$$

Corollary. Let $U \ni 0$ be a connected, open subset of $R^{n}$.

Suppose we have a solution of a Schrödinger operator $(D+V) u=0$ in $U$, $V \in L^{\infty}$ and $\int_{|x|<\varepsilon}|u(x)|^{2} d x=0\left(\varepsilon^{N}\right)$ for any $N$. Then $u \equiv 0$ on $U$.

Theorem 2. Let $n \geq 3, p=(6 n-4) /(3 n+2)$, i.e., $1 / p-1 / 2=1 / \gamma$, with $\gamma=(3 n-2) / 2$. There is a constant $C$ depending only on $n$ such that for all $t \in R$

$$
\begin{gathered}
\left\|e^{t \phi} f\right\|_{L^{2}((-\infty, 0) \times S, d y d w)} \leq C\left\|e^{t \phi} D f\right\|_{L^{p}((-\infty, 0) \times S, d y d w)} \\
\text { for all } f \in C_{0}^{\infty}\left((-\infty, 0) \times S, C^{m}\right) .
\end{gathered}
$$

Moreover,

$$
\begin{gathered}
\left\|e^{t \phi} \nabla f\right\|_{L^{2}((-\infty, 0) \times S, d y d w)} \leq C\left\|e^{t \phi} \Delta f\right\|_{L^{p}((-\infty, 0) \times S, d y d w)} \\
\text { for } f \in C_{0}^{\infty}((-\infty, 0) \times S) .
\end{gathered}
$$

Corollary 2. Let $\Omega$ be a connected, open subset of $R^{n}, n \geq 3$. If $V \in$ $L^{\gamma}(\Omega ; M(m, C))$ and $u$ satisfies $D u \in L^{2}\left(\Omega ; C^{m}\right),(D+V) u=0$ in $\Omega$. If $\int_{|x|<\varepsilon}|u(x)|^{2} d x=0\left(\varepsilon^{N}\right)$ for any $N$, then $u$ is identically zero in $\Omega$.

First, we want to set up some notation and elementary results, following Jerison [4].

2.1. Polar coordinates. Let $S$ denote the unit sphere in $R^{n}$. For $y \in R$ and $w \in S, x=e^{y} w$ gives polar coordinates on $R^{n}$, i.e., $y=\log |x|$ and $w=x /|x|$. The operator

$$
L=\sum_{j<k} \alpha_{j} \alpha_{k}\left(x_{j} \frac{\partial}{\partial x_{k}}-x_{k} \frac{\partial}{\partial x_{j}}\right)
$$

acts only in the $w$-variables $-[L, \partial / \partial y]=0$. We will view $L$ as an operator on the sphere $S$. Let

$$
\hat{\alpha}=\sum_{j=1}^{n} \alpha_{j} x_{j} /|x| \text {. }
$$


Then

$$
\hat{\alpha} D=e^{-y}\left[\frac{\partial}{\partial y}-L\right]
$$

and since $\hat{\alpha}^{2}=-1$,

$$
e^{y} D=\hat{\alpha}\left(\frac{\partial}{\partial y}-L\right) .
$$

Note that $\hat{\alpha}$ is unitary and $L^{*}=L$. If we recall that

$$
\Delta=e^{-2 y}\left(\frac{\partial^{2}}{\partial y^{2}}+(n-2) \frac{\partial}{\partial y}+\Delta_{S}\right),
$$

where $\Delta_{S}$ denotes the Laplace-Beltrami operator of the sphere, then it follows from $D^{*}=D, D^{2}=-\Delta$ that

$$
L(L+n-2)=-\Delta_{S} .
$$

In general if $\psi \in C^{\infty}(R)$, then (3) implies that in polar coordinates $x=e^{y} w$,

$$
e^{t \psi(y)} e^{y} D e^{-t \psi(y)} h=\hat{\alpha} A_{t} h
$$

where $A_{t}=\partial / \partial y-\left(t \psi^{\prime}(y)+L\right)$.

Proof of Theorem 1. To prove the inequality, it suffices to show $A_{t}^{*} A_{t} \geq c t \psi^{\prime \prime}(y)$, since this implies

$$
\left\|A_{t} f\right\|_{L^{2}}^{2}=\left(A_{t}^{*} A_{t} h, h\right) \geq\left(t \psi^{\prime \prime}(y) h, h\right)=t\left\|\sqrt{\psi^{\prime \prime}(y)} h\right\|_{L^{2}(d x)}^{2} .
$$

But

$$
A_{t}^{*} A_{t}=\left(-\frac{\partial}{\partial y}-n-\left(t \psi^{\prime}(y)+L\right)\right)\left(\frac{\partial}{\partial y}-\left(t \psi^{\prime}(y)+L\right)\right) \geq t \psi^{\prime \prime}(y) .
$$

So the claim is true. We had the relation $y=-\psi(y)+e^{-\varepsilon \psi(y)}$. From this, we get

$$
\psi^{\prime}(y)=-1 / 1+\varepsilon e^{-\varepsilon \psi(y)}<0 .
$$

We also find

$$
\psi^{\prime \prime}(y)=\varepsilon^{2} e^{-\varepsilon \psi(y)} /\left(1+\varepsilon e^{-\varepsilon \psi(y)}\right)^{3} \geq c e^{\varepsilon y} .
$$

Now we want to prove Theorem 2.

Proof of Theorem 2. We will prove the following inequality first and prove the dual version later:

$$
\|f\|_{L^{q}\left(e^{n y} d y d w, R^{-} \times S\right)} \leq C\left\|A_{t} f\right\|_{L^{2}\left(e^{n y} d y d w, R^{-} \times S\right)} \quad \text { for } f \in C_{0}^{\infty}(U) .
$$

We can rewrite

$$
A_{t} f=\sum_{k}\left(\frac{\partial}{\partial y}-\left(t \psi^{\prime}(y)+k\right)\right) \pi_{k} f .
$$

If we have an operator of type $\partial / \partial y-a y+b$ for $a, b$ constant coefficients and $a>0$, then we can find a left inverse operator for $\partial / \partial y-a y+b$ easily. So first consider an operator

$$
\Omega=d / d y-y .
$$

Jerison [4] exhibited the following exact formuia for the symbol of a left inverse 
of $\Omega$ : there is a unique operator $B$ on $R$ satisfying $B \Omega=I$ and $\left(B e^{-y^{2} / 2}\right)=0$ given by

$$
B f(y)=(1 / 2 \pi) \int F_{0}(y, \eta) e^{i y \eta} \hat{f}(\eta) d \eta
$$

where

$$
F_{0}(y, \eta)=\sqrt{2} \int_{0}^{\infty} e^{-s^{2}-2 s y} d s e^{-i y \eta-\left(y^{2}+\eta^{2}\right) / 2}-\int_{0}^{\infty} e^{-s^{2}-s(y-i \eta)} d s .
$$

Now if we have an operator $\partial / \partial y-a y+b$, then

$$
\sigma(y, \eta ; a, b)=\frac{1}{\sqrt{a}} F_{0}\left(\sqrt{a} y \delta-\frac{b}{\sqrt{a}}, \frac{\eta}{\sqrt{a}}\right)
$$

is the symbol of the left inverse of $\partial / \partial y-a y+b$.

Then by the method of freezing coefficients, we get an approximate symbol for the inverse of $\partial / \partial y-\left(t \psi^{\prime}(y)+k\right)$. Namely,

$$
F(y, \eta)=\sigma\left(y, \eta ; t \psi^{\prime \prime}(y),-t \psi^{\prime}(y)+t \psi^{\prime \prime}(y) y-k\right) .
$$

Also the following symbol estimate is true:

(8) $\left|\left(\frac{\partial}{\partial y}\right)^{j}\left(\frac{\partial}{\partial}\right)^{l} F_{0}(y, \eta)\right| \leq C_{j, l}(1+|y+i \eta|)^{-1-j-l}, \quad j, l=0,1, \ldots$

From (8) we have similar estimates for our symbol $F(y, \eta)$

$$
\begin{aligned}
& \left|\left(\frac{\partial}{\partial y}\right)^{l}\left(\frac{\partial}{\partial \eta}\right)^{j} F(y, \eta)\right| \\
& \quad \leq C_{j, l}\left(\sqrt{a}+\left|t \psi^{\prime}(y)+k-i \eta\right|\right)^{-1-j-l}\left(a+\left|t \psi^{\prime}(y)+k\right|\right)^{l} .
\end{aligned}
$$

The main tool in the proof is the spherical restriction theorem of Sogge [8].

Theorem. Let $\xi_{k}$ denote the projection operator from $L^{2}(S)$ to the space of spherical harmonics of degree $k$. Then there is a constant $c$ such that

$$
\left\|\xi_{k} g\right\|_{L^{p^{\prime}}(S)} \leq c k^{1-2 / n}\|g\|_{L^{p}(S)}
$$

where $p=2 n /(n+2), p^{\prime}=2 n /(n-2)$. Formula (5) implies that

$$
(L+(n-2) / 2)^{2}=-\Delta_{s}+(n-2)^{2} / 4 \text {. }
$$

Hence

$$
T=\operatorname{sgn}(L+(n-2) / 2)(L+(n-2) / 2)\left(-\Delta_{s}+(n-2)^{2} / 4\right)^{-1 / 2}
$$

is a classical pseudodifferential operator on $S$. Thus $T$ is bounded from $L^{q}\left(S ; C^{m}\right)$ to $L^{q}\left(S ; C^{m}\right)$ for all $q, 1<q<\infty$. Moreover,

$$
\begin{gathered}
\pi_{k}=\frac{1}{2}(1+T) \xi_{k}, \quad k=0,1,2, \ldots, \\
\pi_{k}=\frac{1}{2}(1-T) \xi_{k}, \quad k=1-n,-n,-n-1, \ldots .
\end{gathered}
$$


Therefore, Sogge's theorem implies that

$$
\left\|\pi_{k} g\right\|_{L^{p^{\prime}}\left(S ; C^{m}\right)} \leq C k^{1-2 / n}\|g\|_{L^{p}\left(S ; C^{m}\right)} .
$$

Define $\pi_{M, N}$ by

$$
\pi_{k} \pi_{M, N} g=\left\{\pi_{k} g \text { if } M \leq k \leq N, 0 \text { otherwise }\right\} .
$$

The triangle inequality implies

$$
\left\|\pi_{M, N} g\right\|_{L^{p^{\prime}}\left(S ; C^{m}\right)} \leq C N^{1-2 / n}(N-M+1)\|g\|_{L^{p}\left(S ; C^{m}\right)} .
$$

Next use a device due to Tomas [11]:

$$
\begin{aligned}
\left\|\pi_{M, N} g\right\|_{L^{2}}^{2} & =\int_{S}\left\langle\pi_{M, N} g, g\right\rangle \leq\left\|\tau_{M, N} g\right\|_{L^{p^{p}}}\|g\|_{L^{p}} \\
& \leq C N^{1-2 / N}(N-M+1)\|g\|_{L^{p}}^{2} .
\end{aligned}
$$

We conclude that

$$
\left\|\pi_{M, N} g\right\|_{L^{2}\left(S ; C^{m}\right)} \leq C N^{1 / p^{\prime}}(N-M+1)^{1 / 2}\|g\|_{L^{p}\left(S ; C^{m}\right)}
$$

and by duality

$$
\left\|\pi_{M, N} g\right\|_{L^{p^{\prime}}\left(S ; C^{m}\right)} \leq C N^{1 / p^{\prime}}(N-M+1)^{1 / 2}\|g\|_{L^{2}\left(S ; C^{m}\right)} .
$$

If we interpolate with the trivial estimate

$$
\left\|\pi_{M, N} g\right\|_{L^{2}\left(S ; C^{m}\right)} \leq\|g\|_{L^{2}\left(S ; C^{m}\right)}
$$

we find that

$$
\left\|\pi_{M, N} g\right\|_{L^{q}\left(S ; C^{m}\right)} \leq C\left(N^{(n-2) / 2}(N-M+1)^{n / 2}\right)^{1 / 2-1 / q}\|g\|_{L^{2}\left(S ; C^{m}\right)}
$$

for $2 \leq q \leq p^{\prime}=2 n / n-2$.

Let $N$ be the integer satisfying $2^{N-1} \leq 10 e^{\varepsilon j / 2} t^{1 / 2} \leq 2^{N}$. Consider a partition of unity $\left\{\phi_{\beta}\right\}_{\beta=0}^{N}$ of the positive real axis satisfying

$$
\sum_{\beta=0}^{N} \phi_{\beta}(r)=1, \quad \text { all } r>0,
$$

$$
\begin{aligned}
\operatorname{supp} \phi_{\beta} \subset\left\{r: 2^{\beta-2} \leq r \leq 2^{\beta}\right\}, \quad \beta & =1,2, \ldots, N-1, \\
\operatorname{supp} \phi_{0} & \subset\{r: r \leq 1\}, \quad \operatorname{supp} \phi_{N} \subset\{r: r \geq s / 400\},
\end{aligned}
$$

$$
\left|(\partial / \partial r)^{l} \phi_{\beta}(r)\right| \leq C_{l} 2^{-\beta l}, \quad l=0,1, \ldots .
$$

Define

$$
F_{t} f(y, w)=\sum_{k} \frac{1}{2 \pi} \int F_{t}(y, \eta, k) \pi_{k} \tilde{f}(\eta, \cdot)(w) e^{i \eta y} d \eta
$$

Define

$$
F_{t}^{\beta}(y, \eta, k)=\phi_{\beta}\left(\frac{1}{\sqrt{a}}\left|t \psi^{\prime}(y)+k-i \eta\right|\right) F_{t}(y, \eta, k) .
$$


Then $F_{t}$ satisfies

$$
\begin{aligned}
& \left|\left(\frac{\partial}{\partial \eta}\right)^{j}\left(\frac{\partial}{\partial y}\right)^{l} F_{t}(y, \eta, k)\right| \\
& \quad \leq C_{j, l}\left(\sqrt{a}+\left|t \psi^{\prime}(y) \delta+k-i \eta\right|\right)^{-1-j-l}\left(a+\left|t \psi^{\prime}(y)+k\right|\right)^{l}, \\
& \left|\left(\frac{\partial}{\partial \eta}\right)^{j}\left(F_{t}(y, \eta, k)-F_{t}(y, \eta, k+1)\right)\right| \\
& \quad \leq C_{j}\left(\sqrt{a}+\left|t \psi^{\prime}(y)+k-i \eta\right|\right)^{-2-j}
\end{aligned}
$$

From (12) and the property of $\left|(\partial / \partial \delta r)^{l} \phi_{\beta}(r)\right| \leq 2^{-\beta l}$, we deduce that the following inequalities hold uniformly for $y \in I=I_{l}=(-l,-l+1)$

$$
\begin{gathered}
\left|\left(\frac{\partial}{\partial \eta}\right)^{j} F_{t}^{\beta}(y, \eta, k)\right| \leq C_{j}\left(2^{\beta} \sqrt{a}\right)^{-1-j}, \\
\left|\left(\frac{\partial}{\partial \eta}\right)^{j}\left(F_{t}^{\beta}(y, \eta, k)-F_{t}^{\beta}(y, \eta, k+1)\right)\right| \leq C_{j}\left(2^{\beta} \sqrt{a}\right)^{-2-j} .
\end{gathered}
$$

Now if we define

$$
\left(F_{t}^{\beta} f\right)(y, w)=\sum_{k} \frac{1}{2 \pi} \int F_{t}^{\beta}(y, \eta, k) \pi_{k} \tilde{f}(\eta, \cdot)(w) e^{i y \eta} d \eta,
$$

then $F_{t}=\sum_{\beta=0}^{N} F_{t}^{\beta}$. We begin by estimating $F_{t}^{N}$. In the case $\beta=N$, we need different estimates. By a choice of $N$ such that $2^{N} \sim 10 e^{\varepsilon j} \sqrt{a}$ we have the following.

Since $F_{t}^{N}$ is supported where

$$
\left|t \psi^{\prime}(y)+k-i \eta\right| \geq 2^{N} \sqrt{a} \sim 10 t
$$

we have

$$
\left|t \psi^{\prime}(y)+k-i \eta\right|>c(1+|\eta|+|k|) \quad \text { uniformly for } y<0 \text {. }
$$

Hence

$$
\left|\left(\frac{\partial}{\partial \eta}\right)^{j}\left(\frac{\partial}{\partial y}\right)^{m} F_{t}^{N}(y, \eta, k)\right| \leq C_{j, m}(1+|\eta|+|k|)^{-1-j} \delta, \quad j=0,1, \ldots
$$

It follows that $F_{t}^{N}$ is controlled by standard pseudodifferential operators and by the Sobolev inequality

$$
\|f\|_{L^{p^{\prime}\left(I \times S, e^{n y} d y d w\right)}} \leq\|r D f\|_{L^{2}\left(I \times S, e^{n y} d y d w\right)}, \quad \text { for all } f \in C_{0}^{\infty}(I \times S)
$$

and $p^{\prime}=2 n /(n-2)$.

We have

$$
\left\|F_{t}^{N} f\right\|_{L^{q}(I \times S, d x)} \leq C\|f\|_{L^{2}(I \times S, d x)}
$$

for all $f \in C_{0}^{\infty}\left(I \times S ; C^{m}\right)$ for $1 \leq q \leq p^{\prime}$. In particular this holds for $q=(6 n-4) /(3 n-6)$.

Let

$$
M=\left[-t \psi^{\prime}(y)-2^{\beta} \sqrt{a}\right], \quad M^{\prime}=\left[M+2 \times 2^{\beta} \sqrt{a}\right]+1 .
$$


Denote

$$
T_{t}^{\beta}(y, \eta) g(w)=\sum_{k} F_{t}^{\beta}(y, \eta, k) \pi_{k} g(w) .
$$

Here $F_{t}^{\beta}(y, \eta, k)=0$ unless $M \leq k \leq M^{\prime}$. Summation by parts gives

$$
T_{t}^{\beta}(y, \eta)=\sum_{M}^{M^{\prime}}\left(F_{t}^{\beta}(y, \eta, k)-F_{t}^{\beta}(y, \eta, k+1)\right) \pi_{M, k} \quad \text { for } M \leq k \leq M^{\prime} .
$$

Now (10) and (13) give

$$
\begin{aligned}
& \left\|\left(\frac{\partial}{\partial \eta}\right)^{j} T_{t}^{\beta}(y, \eta) \pi_{M, k} g\right\|_{L^{q}\left(S ; C^{m}\right)} \\
& \quad \leq C_{j}\left(2^{\beta} \sqrt{a}\right)^{-1-j}\left(t^{(n-2) / 2}\left(2^{\beta} \sqrt{a}\right)^{n / 2}\right)^{1 / 2-1 / q}\|g\|_{L^{2}\left(S ; C^{m}\right)}
\end{aligned}
$$

uniformly for $y \in I$.

Define

$$
\begin{aligned}
K_{t}^{\beta}(y, z) & =\frac{1}{2 \pi} \int T_{t}^{\beta}(y, \eta) e^{i z \eta} d \eta \\
& =\frac{1}{2 \pi} \int\left(\frac{\partial}{\partial \eta}\right)^{j} T_{t}^{\beta}(y, \eta) 1 /(i z)^{j} e^{i z \eta} d \eta
\end{aligned}
$$

and since the length of the interval in $\eta$ where $T_{t}^{\beta}$ is nonzero is less than $2 \times 2^{\beta} \sqrt{a}$,

$$
\begin{aligned}
& \left\|K_{t}^{\beta}(y, z) g\right\|_{L^{q}\left(S ; C^{m}\right)} \\
& \quad \leq C\left(1+\left|2^{\beta} \sqrt{a} z\right|\right)^{-10}\left(t^{(n-2) / 2}\left(2^{\beta} \sqrt{a}\right)^{n / 2}\right)^{1 / 2-1 / q}\|g\|_{L^{2}\left(S ; C^{m}\right)} .
\end{aligned}
$$

Note that

$$
F_{t}^{\beta} f(y, w)=\int K_{t}^{\beta}\left(y, y-y^{\prime}\right) f\left(y^{\prime}, \cdot\right)(w) d y^{\prime} .
$$

Lemma. Let $H\left(y, y^{\prime}\right)$ be a bounded operator from $L^{p}(S)$ to $L^{q}(S)$ of operator norm $\leq h\left(y-y^{\prime}\right)$ for each $y, y^{\prime} \in R$. Suppose that $h \in L^{r}(R)$ for $1 / r+1 / p=$ $1+1 / q$. Then

$$
T f(y, w)=\int H\left(y, y^{\prime}\right) f\left({ }^{\prime}:\right)(w) d y^{\prime}
$$

satisfies

$$
\|T f\|_{L^{q}(R \times S)} \leq\|h\|_{L^{r}(R)}\|f\|_{L^{p}(R \times S)} .
$$

Now we see that the lemma implies for $\beta \leq N-1$,

$$
\left\|F_{t}^{\beta} f\right\|_{L^{q}\left(I_{j} \times S, d x\right)} \leq C 2^{-(n-2) \beta / \gamma}\left(e^{\varepsilon y}\right)^{m}\|f\|_{L^{2}\left(I_{j} \times S, d x\right)}, \quad m>-1 / 3 .
$$

If we sum the series in $\beta$ and add the final term $\beta=N$, we get

$$
\left\|\sum_{\beta} F_{t}^{\beta} f\right\|_{L^{q}\left(I_{j} \times S, e^{n y} d y d w\right)} \leq C^{\prime} e^{\varepsilon j / 3}\|f\|_{L^{2}\left(I_{j} \times S, e^{n y} d y d w\right)} \cdot
$$

So far we have obtained estimates only for the main term, so we will work on the remainder term from now on. 
From the relation $f(y)=F_{t} A_{t} f(y)-R_{t} f(y)$, we have

$$
R_{t} f(y)=\iint F_{t}(y, \eta, k) t\left(y-y^{\prime}\right)^{2} g\left(y, y^{\prime}\right) e^{i\left(y-y^{\prime}\right) \eta} f\left(y^{\prime}, \cdot\right)(w) d y^{\prime} d \eta \pi_{k}
$$

for

$$
g\left(y, y^{\prime}\right)=\int_{0}^{1}(1-s) \psi^{\prime \prime \prime}(y)\left(y+s\left(y^{\prime}-y\right)\right) d s .
$$

We hope to obtain a similar type of inequality, i.e.,

$$
\left\|R_{t} f\right\|_{L^{q}\left(I_{l} \times S, d x\right)} \leq C_{l}\|f\|_{L^{2}\left(I_{l} \times s, d x\right)} \quad \text { in } I_{l}=(-l,-l+1) .
$$

For that we are going to adopt the same techniques as in the main step. Then using the same partition of unity, $\left\{\phi_{\beta}\right\}_{\beta=0, \ldots, N}$,

$$
R_{t}^{\beta} f(y)=\sum \iint F_{t}^{\beta}(y, \eta, k) t\left(y-y^{\prime}\right)^{2} e^{i \eta\left(y-y^{\prime}\right)} g\left(y, y^{\prime}\right) f\left(y^{\prime}, \cdot\right)(w) d y^{\prime} d \eta \pi_{k} .
$$

Let's denote $\widetilde{K}_{t}^{\beta}\left(y, y^{\prime}\right)$ as follows:

$$
\widetilde{K}_{t}^{\beta}\left(y, y^{\prime}\right)=\frac{1}{2 \pi} \int t T_{t}^{\beta}(y, \eta)\left(y-y^{\prime}\right)^{2} e^{i\left(y-y^{\prime}\right) \eta} g\left(y, y^{\prime}\right) d \eta .
$$

Then the following relation holds:

$$
R_{t}^{\beta} f(y, w)=\int \widetilde{K}_{t}^{\beta}\left(y, y^{\prime}\right) f\left(y^{\prime}, \cdot\right)(w) d y^{\prime} .
$$

Since $a=t \psi^{\prime \prime}(y) \sim t e^{-\varepsilon l}$ uniformly for $y \in I_{l}$ and $\|g\|_{\infty} \leq e^{-\varepsilon l}$ for $y$, $y^{\prime} \in I_{l}$.

As a result, $\left\|t\left(2^{\beta} \sqrt{a}\right)^{-2} g\right\|_{\infty} \leq C^{\prime}$. Then following the same steps as before we obtain

$$
\left\|R_{t}^{\beta} f\right\|_{L^{q}\left(I_{l} \times S, d x\right)} \leq C^{\prime} 2^{-\beta(n-2 / \gamma)} e^{\varepsilon l / 3}\|f\|_{L^{2}\left(I_{l} \times S, d x\right)} \quad \text { when } \gamma=(3 n-2) / 2 .
$$

The case $\beta=N$ works for the same reason as in the main terms: from the definition,

$$
\left(R_{t}^{n} f\right)(y, w)=\sum_{k} \frac{1}{2 \pi} \iint \widetilde{F}_{t}^{N}\left(y, y^{\prime}, \eta, k\right) \pi_{k} f\left(y^{\prime}, \cdot\right)(w) e^{i \eta\left(y-y^{\prime}\right)} d y^{\prime} d w
$$

when $\widetilde{F}_{t}^{N}\left(y, y^{\prime}, \eta, k\right)=\left(y-y^{\prime}\right)^{2} g\left(y, y^{\prime}\right) F_{t}^{N}$.

Now as in the main step this operator is controlled by standard pseudodifferential operators and we can deduce

$$
\left\|\widetilde{F}_{t}^{N} f\right\|_{L^{q}\left(I_{l} \times S, d x\right)} \leq C\|f\|_{L^{2}\left(I_{l} \times S, d x\right)} .
$$

If we sum the series in $\beta$ and add the final term $\beta=N$, we obtain

$$
\left\|R_{t} f\right\|_{L^{q}\left(I_{l} \times S, d x\right)} \leq C e^{\varepsilon \delta j / 3}\|f\|_{L^{2}\left(I_{l} \times S, d x\right)} .
$$

Now with the estimate on the unit anulus, i.e. $I_{l} \times S$, we try to extend it to the whole ball, in this case $R^{-} \times S$. First, we restate Theorem 1 as

$$
\|f\|_{L^{2}\left(I_{j} \times S, e^{n y} d y d w\right)} \leq C e^{\varepsilon j / 2}\left\|A_{t} f\right\|_{L^{2}\left(I_{j} \times S, e^{n y} d y d w\right)} .
$$

Combining this with $\left(14^{\prime}\right)$ we obtain

$$
\left\|R_{t} f\right\|_{L^{q}\left(I_{j} \times S, e^{n y} d y d w\right)} \leq C e^{5 \varepsilon j / 6}\left\|A_{t} f\right\|_{L^{2}\left(I_{j} \times S, e^{n y} d y d w\right)}
$$


and

$$
\|f\|_{L^{q}\left(I_{j} \times S, e^{n y} d y d w\right)} \leq C e^{5 \varepsilon j / 6}\left\|A_{t} f\right\|_{L^{2}\left(I_{j} \times S, e^{n y} d y d w\right)} .
$$

But with the main estimates, the above implies

$$
\|f\|_{L^{q}\left(I_{j} \times S, e^{(4 \varepsilon q / 3+n) y} d y d w\right)}^{q} \leq C\left\|A_{t} f\right\|_{L^{2}\left(I_{j} \times S, e^{(n+\varepsilon) y} d y d w\right)}^{q} .
$$

Now choose $\left\{\psi_{j k}\right\}_{j \in N, k=1,2}$ to be partitions of unity such that

$$
\psi_{j 1} \in C_{0}^{\infty}(-j,-j+3 / 4), \quad \psi_{j 2} \in C_{0}^{\infty}(-j+2 / 4,-j+5 / 4) .
$$

Then using $f=\sum \psi_{j} f$ (we will just call $\left\{\psi_{j k}\right\},\left\{\psi_{j}\right\}$ ), we come to the final estimate:

$$
\begin{aligned}
& \|f\|_{L^{q}\left(e^{(4 e q / 3+n) y} d y d w, R^{-} \times S\right)}^{q} \\
& \quad \leq{ }^{(1)} C_{0} \sum_{j}\left\|\psi_{j} f\right\|_{L^{q}\left(e^{(4 e q / 3+n) y} d y d w, I_{j} \times S\right)}^{q} \\
& \quad \leq C_{1} \sum_{j}\left\|A_{t}\left(\psi_{j} f\right)\right\|_{L^{2}\left(e^{(n+\varepsilon) y} d y d w, I_{j} \times S\right)}^{q} \\
& \quad \leq C_{2} \sum_{j}\left\|\psi_{j}^{\prime} f\right\|_{L^{2}\left(e^{(n+e) y} d y d w, I_{j} \times S\right)}^{q}+C_{2} \sum_{j}\left\|\psi_{j} A_{t} f\right\|_{L^{2}\left(e^{(n+e) y} d y d w, I_{j} \times S\right)}^{q} \\
& \quad \leq{ }^{(2)} C^{\prime}\|f\|_{L^{2}\left(e^{(n+\varepsilon) y} d y d w, R^{-} \times S\right)}^{q}+C^{\prime}\left\|A_{t} f\right\|_{L^{2}\left(e^{(n+\varepsilon) y} d y d w, R^{-} \times S\right)}^{q} \\
& \quad \leq{ }^{(3)} C^{\prime \prime}\left\|A_{t} f\right\|_{L^{2}\left(e^{n y} d y d w, R^{-} \times S\right)}^{q} .
\end{aligned}
$$

Inequalities (1) and (2) hold since for each $x \in R$, only finitely many $\psi_{j}$ 's overlap. Inequality (3) comes from $L^{2}$ estimates. The above estimate is equivalent to

$$
\left\|e^{t \psi} f\right\|_{L^{q}\left(R^{-} \times S, e^{n y} d y d w\right)} \leq C\left\|e^{t \psi} D f\right\|_{L^{2}\left(R^{-} \times S, e^{n y} d y d w\right)} .
$$

\section{REFERENCES}

1. S. Alinhac and M. S. Baouendi, Uniqueness for the characteristic Cauchy problem and strong unique continuation for high order P.D.E., Amer. J. Math. 102 (1980).

2. T. Carleman, Sur un problèm d'unicité pour les systèmes d'èquations aux derivées partielles à deux variables indépendantes, Ark. Mat. 26B (1939), 1-9.

3. L. Hörmander, Uniqueness theorems for second order elliptic partial differential equations, Comm. Partial Differential Equations 8 (1983), 21-63.

4. D. Jerison, Carleman inequalities for the Dirac and Laplace operators and unique continuation, Adv. Math. 62 (1986).

5. D. Jerison and C. E. Kenig, Unique continuation and absence of positive eigenvalues for Schrödinger operators, Ann. of Math. (2) 121 (1985).

6. C. E. Kenig, Uniform Sobolev inequalities for second order differential operators and unique continuation theorem, Internat. Congr. Math., 1986.

7. B. Simon, Schrödinger semigroups, Bull. Amer. Math. Soc. (N.S.) 7 (1982), 447-526.

8. C. Sogge, Oscillatory integrals and spherical harmonics, Thesis, Princeton University, Princeton, NJ, 1985.

9. E. M. Stein, Singular integrals and differentiability properties of functions, Princeton Univ. Press, Princeton, NJ, 1970.

10. M. E. Taylor, Pseudo differential operators, Princeton Univ. Press, Princeton, NJ, 1981. 
11. P. Tomas, A restriction theorem for the Fourier transform, Bull. Amer. Math. Soc. (N.S.) 81 (1975), 477-478.

12. T. Wolff, Note on counter examples in strong unique continuation problems, Proc. Amer. Math. Soc. 114 (1992), 351-356.

Department of Science, School of Engineering, Hong Ik University, 72-1 Mapogu, SaNgsudong, SeOUl, 121-791 Korea 